\title{
RECURSO DE TECNOLOGIA ASSISTIVA PARA ESTUDANTES COM PARALISIA CEREBRAL: O JOGO DA VELHA COMO MODELO METODOLÓGICO
}

Manoel Osmar Seabra Junior, Franciele Aparecida dos Santos Felicio, Viviane Rodrigues, Talita Maria Souza Santos

Universidade Estadual Paulista - UNESP, Presidente Prudente, SP.

Agência de fomento: PIBIC-Cnpq

\section{RESUMO}

O objetivo deste estudo foi avaliar os efeitos do jogo da velha adaptado sobre o desenvolvimento e ampliação das habilidades manipulativas de três crianças com paralisia cerebral com idades de 10 a 12 anos. Para tanto, utilizou-se como metodologia o delineamento de linha de base múltipla por sujeitos, que tem por finalidade analisar, simultaneamente, mais de uma variável dependente. Desse modo, os resultados encontrados denotam-se para a utilização favorável desse recurso direcionado para a vida dessas crianças, por se tratar de um jogo que traz características que auxiliam e estimulam as habilidades manipulativas e, consequentemente, para a melhora nas atividades de vida diária das mesmas. Conclui-se que a utilização do jogo da velha como um recurso para interação e inclusão de crianças com paralisia cerebral pode ser vista como uma ferramenta eficaz para atender as diversas características e auxiliando nas atividades escolares, de lazer e de vida diária.

Palavras-chave: Educação Especial. Paralisia Cerebral. Jogo da Velha. Tecnologia Assistiva.

\section{ASSISTIVE TECHNOLOGY RESOURCE FOR STUDENTS WITH CEREBRAL PARALYSIS: NOUGHTS AND CROSSES AS A METHODOLOGICAL MODEL}

\begin{abstract}
:
The purpose of this study was to evaluate the effects of the adapted game of de old on the development and expansion of the manipulative abilities of three children with cerebral palsy aged 10 to 12 years. For this, the multiple baseline design by subjects was used as the methodology, whose purpose is to simultaneously analyze more than one dependent variable. Thus, the results show the favorable use of this resource for the life of these children, because it is a game that has characteristics that help and stimulate the manipulative skills and consequently to improve their daily life activities. It concludes that the use of the game of the old as a resource for interaction and inclusion of children with Cerebral Palsy can be seen as an effective tool to meet the various characteristics and helping in the difficulties encountered in the activities of school, leisure and daily living.
\end{abstract}

Keywords: Special Education. Cerebral Palsy. Noughts and crosses. Assistive Technology 


\section{INTRODUÇÃO}

A pessoa com paralisia cerebral, de acordo com as Diretrizes de Atenção à Pessoa com Paralisia Cerebral (BRASIL, 2013), apresenta uma "desordem motora que pode, frequentemente, vir acompanhada por distúrbios sensoriais, perceptivos, cognitivos, de comunicação e comportamental; epilepsia e problemas musculoesqueléticos secundários" (ROSENBAUM et al., 2007, p. 27).

Não obstante, ao receber oportunidades de interação por meio de jogos, estes podem proporcionar a utilização de diversas habilidades, tais como motoras, cognitivas, de linguagem e atenção (PERES, 2004; LAMÔNACO; CAZEIRO, 2006; BRUNO, 2009; BRASIL, 2013). Nesta direção, o foco é a estimulação para que a criança interaja com o meio e com os objetos e, por sua vez, conheça, reconheça e discrimine pessoas, locais e objetos com destreza motora, capacidades sensoriais e perceptivas.

O jogo da velha é um jogo com regras simples, porém demanda diversas habilidades, dentre elas, as habilidades motoras como preensão palmar e movimentos precisos para colocar as peças nos lugares desejados pelos jogadores. Desta forma, considerando as pessoas com paralisia cerebral, sabe-se que há, em alguns casos, comprometimento motor (LAMÔNICA et al., 2003) e este deve ser considerado no momento de interação com o jogo. Assim, as adaptações devem ser realizadas para que as habilidades motoras sejam potencializadas, favorecendo o jogador com paralisia cerebral durante o jogo.

Neste sentido, um jogo que possibilite à pessoa a superação de suas limitações e ofereça igualdade de oportunidades, caracteriza-se como um recurso de Tecnologia Assistiva, visto que promove a funcionalidade da pessoa com paralisia cerebral, visando a sua independência e inclusão (CAT- Comitê de Ajudas Técnicas, 2007).

Destarte, o objetivo do presente estudo foi avaliar os efeitos do jogo da velha adaptado sobre o desenvolvimento e ampliação das habilidades manipulativas de três crianças com paralisia cerebral com idades de 10 a 12 anos.

\section{METODOLOGIA}

Seguindo os cumprimentos éticos para a realização da pesquisa foram realizados os seguintes procedimentos, coleta das assinaturas dos responsáveis no termo de consentimento livre e esclarecido (TCLE). Os responsáveis pelo estudante, autorizaram sua participação, que se encontra registrado sob o CAEE 94784218800005402 do Comitê de Ética em Pesquisa (CEP).

\section{Participantes e Local}

Os participantes do estudo foram três estudantes com o diagnóstico de encefalopatia crônica progressiva (paralisia cerebral). Com idades variadas, porém com característica próximas. O Participante 1 (P1): sexo masculino 11 anos de idade, cadeirante e com dificuldade motora fina comprometida, atrofia nos membros superiores, estudante de uma entidade para crianças com paralisia cerebral. O Participante 2 (P2): sexo feminino, 10 anos de idade, cadeirante, motricidade fina e global comprometidas, dificuldade com a fala, atrofia membros superiores e movimentos espásticos, estudante de uma entidade para crianças com paralisia cerebral. O Participante 3 (P3): sexo feminino, 12 anos de idade, estudante do ensino regular, cadeirante, atrofia de membros superiores, dificuldades de motricidade global e fina.

Os atendimentos ocorreram uma vez na semana, com duração de 1 hora, no interior do laboratório de pesquisa de uma universidade e em uma sala de aula específica disponibilizada pela instituição. 


\section{MATERIAIS E EQUIPAMENTOS}

O jogo da velha foi o jogo escolhido para a pesquisa por ser jogo tradicional e competitivo que desperta um interesse maior pelos participantes, além de fazer com que o jogador utilize a motricidade fina e global, podendo desenvolver habilidades e competências importantes para as atividades de vida diária desses participantes.

No Quadro 1, apresentam-se as características do jogo pré-adaptado e das adaptações realizadas para a intervenção, assim como suas descrições:

Quadro 1. Jogo tradicional (pré-adaptado) e com adaptação para a intervenção

Possui um
tabuleiro de
$30 \times 30 \mathrm{~cm}$ feito
de material
flexível
plástico,
possui
peças de EVA
(5 círculos e 5
x).
quasterísticas do

Fonte da Imagem esquerda no quadro: Jogo pré-adaptado extraída de: http://marioramaobenevides.blogspot.com. Fonte da Imagem central no quadro: Jogo adaptado: elaboração própria, 2018.

O jogo da velha foi adaptado de forma a proporcionar melhor identificação das casas e melhor posicionamento das peças. Foi colocado velcro nas peças e nas casas do jogo para identificar cada casa. Foi também colocado em torno de cada quadrado (casas) velcro para que possam senti-los por meio do tato.

\section{Variáveis do estudo e Procedimento de Coleta e Análise dos Dados}

Foram utilizados, para coleta e análise de dados, uma folha de registro das variáveis de respostas com pontuações de 0 a 3 pontos, demonstrando sua porcentagem alcançada em cada sessão da pesquisa, conforme apresentado no Quadro 2: 
Quadro 2. Variáveis Dependentes e suas respectivas descrições

\begin{tabular}{|c|c|c|c|c|c|}
\hline \multicolumn{6}{|c|}{ Variáveis Dependentes (VD) } \\
\hline Variáveis & & $\begin{array}{l}\text { Precisão do } \\
\text { ovimento/ } \\
\text { sicionamento }\end{array}$ & $\begin{array}{l}\text { 2. Amplitude } \\
\text { movimento }\end{array}$ & $\begin{array}{lr}\text { 3. } & \text { Capacidade } \\
\text { de } & \text { preensão } \\
\text { palmar }\end{array}$ & $\begin{array}{l}\text { 4. Velocidade } \\
\text { do } \text { Movimento }\end{array}$ \\
\hline \multirow[t]{2}{*}{$\begin{array}{l}\text { Descrição } \\
\text { da } \\
\text { variável }\end{array}$} & \multicolumn{2}{|c|}{$\begin{array}{ll}\text { Precisão } & \text { do } \\
\text { movimento e do } \\
\text { posicionamento } \\
\text { das peças no } \\
\text { tabuleiro }\end{array}$} & $\begin{array}{l}\text { Amplitude do } \\
\text { movimento } \\
\text { executado, por } \\
\text { exemplo levantar as } \\
\text { mãos para mover as } \\
\text { peças }\end{array}$ & $\begin{array}{l}\text { Se consegue } \\
\text { segurar as } \\
\text { peças }\end{array}$ & $\begin{array}{ll}O \quad \text { tempo } & d \epsilon \\
\text { execução } & d c \\
\text { movimento } & \end{array}$ \\
\hline & & $\begin{array}{l}\text { Não executa o } \\
\text { movimento }\end{array}$ & $\begin{array}{l}\text { Não executa } \\
\text { movimento }\end{array}$ & $\begin{array}{l}\text { Não executa o } \\
\text { movimento }\end{array}$ & $\begin{array}{l}\text { Não executa } 0 \\
\text { movimento }\end{array}$ \\
\hline \multirow[t]{3}{*}{ Pontuação } & 1 & $\begin{array}{l}\text { Executa com } \\
\text { assistência física }\end{array}$ & $\begin{array}{l}\text { Executa com } \\
\text { assistência física }\end{array}$ & $\begin{array}{l}\text { Executa com } \\
\text { assistência } \\
\text { física }\end{array}$ & $\begin{array}{l}\text { Executa } \\
\text { lentamente }\end{array}$ \\
\hline & 2 & $\begin{array}{l}\text { Executa com } \\
\text { dificuldade, mas } \\
\text { sozinho }\end{array}$ & $\begin{array}{l}\text { Executa } \\
\text { dificuldade, } \\
\text { sozinho }\end{array}$ & $\begin{array}{l}\text { Executa com } \\
\text { dificuldade, } \\
\text { mas sozinho }\end{array}$ & $\begin{array}{l}\text { Executa } \\
\text { moderadamente }\end{array}$ \\
\hline & & $\begin{array}{l}\text { Executa com } \\
\text { independência, } \\
\text { sem } \\
\text { dificuldades }\end{array}$ & $\begin{array}{|ll|}\text { Executa } & \text { com } \\
\text { independência, } & \text { sem } \\
\text { dificuldades } & \\
\end{array}$ & $\begin{array}{l}\text { Executa com } \\
\text { independência, } \\
\text { sem } \\
\text { dificuldades }\end{array}$ & $\begin{array}{l}\text { Executa } \\
\text { rapidamente }\end{array}$ \\
\hline
\end{tabular}

Fonte: elaboração própria, 2018.

Com este procedimento, pode-se ter um controle tanto das etapas de linha de base como intervenção que ocorreram da seguinte forma:

Linha de Base: os três participantes iniciaram a linha de base juntos, cada participante executava 5 tentativas do jogo em cada sessão, ou seja, foi disposto o jogo e eles podiam executar ou não a interação com o jogo. Não foi explicado regras, uma vez que a intenção não era o jogo em si, mas as habilidades manipulativas que por meio dele os participantes iriam necessitar para jogá-lo. As sessões tinham duração de aproximadamente 50 minutos, foram realizadas três sessões para P1, cinco sessões para P2 e seis sessões para P3.

Intervenção: após a estabilidade da linha de base de $\mathrm{P} 1$, os demais permaneceram em linha de base, dando-se início à intervenção com o P1. Quando P1 atingiu o critério de 75\%, iniciou-se a intervenção com P2. Quando P2 atingiu o critério, iniciou-se a intervenção com P3. Assim, a intervenção consistia no oferecimento do Jogo da Velha Adaptado para crianças com paralisia cerebral e os efeitos deste jogo sobre as habilidades manipulativas dos participantes. Nesta fase foram realizadas três sessões para P1, quatro sessões para P2 e cinco sessões para P3.

\section{DELINEAMENTO EXPERIMENTAL}

Este estudo caracteriza-se por uma pesquisa experimental por meio da utilização do delineamento de linha de base múltipla que consistem em oferecer um controle experimental, dividindo-se em linha de base e intervenção.

A linha de base é estabelecida para todos os participantes. Inicia-se a intervenção com o primeiro participante quando a linha de base estiver estável em tendência e nível, enquanto os outros participantes ficam em linha de base. Quando o primeiro participante atinge o critério de desempenho, inicia-se a intervenção com o segundo participante que está em linha de base, assim que o segundo participante atinge o critério de desempenho, inicia-se a intervenção com o 
terceiro participante que está em linha de base e assim sucessivamente. Em síntese a intervenção é iniciada em diferentes momentos para cada participante.

A linha de base, de acordo com Cozby (2003), é a mensuração do comportamento antes do período de manipulação das variáveis, sendo ela importante para que o pesquisador consiga avaliar o desempenho do aluno quando ocorrer a introdução do treinamento. Já a intervenção é a etapa em que ocorre a introdução do treinamento, onde o pesquisador estimula o participante, por meio de estratégias e adaptações dos jogos.

\section{RESULTADOS}

Para alcançar os resultados obtidos foram necessárias as etapas de linha de base, de intervenção e follow-up como apresentado anteriormente, isso para se ter um feedback positivo do recurso utilizado. Os dados foram apresentados em forma gráfica conforme demonstrado abaixo na figura 1, assim como as adaptações realizadas durante a etapa de linha de base, para aprimorar o recurso e o desempenho dos participantes. Essas adaptações foram necessárias uma vez que se notou uma dificuldade em manter as peças no lugar devido as dificuldades motoras e de localização correta das casas.

Figura 1. Precisão do Movimento/ Posicionamento e Amplitude de movimento
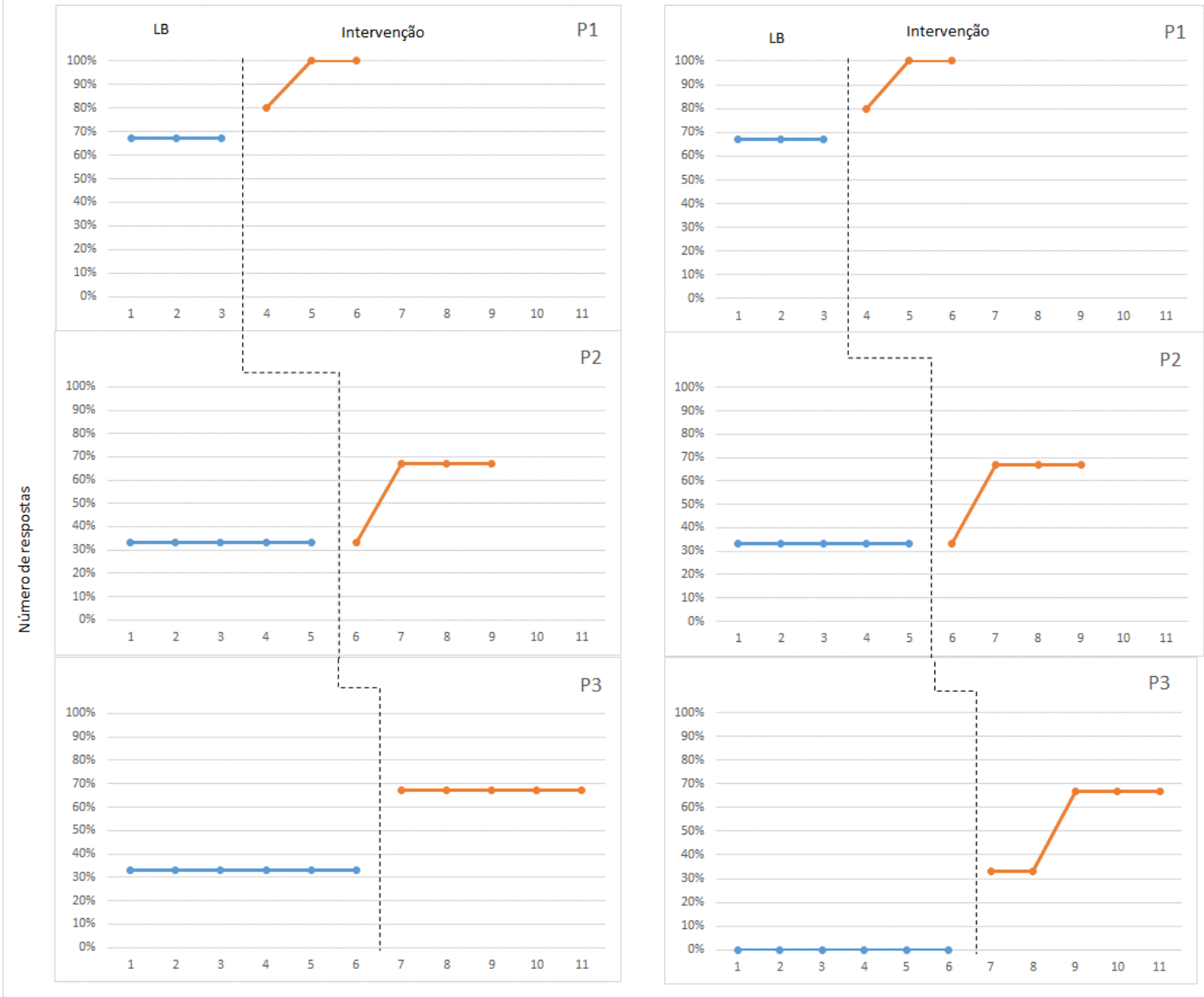

Sessőes

Fonte: elaboração própria, 2018.

Conforme a figura 1, observa-se que na linha de base P1 obteve um desempenho satisfatório, devido ao fato do participante ter boas condições motoras dos membros superiores. Nas três sessões de intervenção o participante teve uma média de $93 \%$, demonstrando um 
aumento de $26 \%$ da linha de base. Com relação ao P2, este obteve um desempenho de $33 \%$ na linha de base, na intervenção alcançou uma média de 59\%, compondo um aumento de $26 \%$.

Considerando o desempenho de P3, observa-se que em linha de base este aluno obteve uma pontuação de $33 \%$. Após a intervenção obteve uma média de $67 \%$, compondo um aumento de $34 \%$ em seu desempenho. Assim, conclui-se que os três participantes aumentaram seus desempenhos após a introdução da intervenção.

Quanto à amplitude do movimento, observa-se que P1 obteve $67 \%$ de desempenho na linha de base e $93 \%$ na intervenção, atingindo uma média de $26 \%$. Com relação ao P2, este obteve um desempenho de 33\% na linha de base e na intervenção alcançou uma média de 59\%, compondo um aumento de $26 \%$. Em continuidade, P3 não obteve nenhuma pontuação em linha de base, atingindo uma média de 53\%, em suma, obteve um aumento significativo após as adaptações realizadas no jogo.

Figura 2. Capacidade de preensão palmar e Velocidade do Movimento

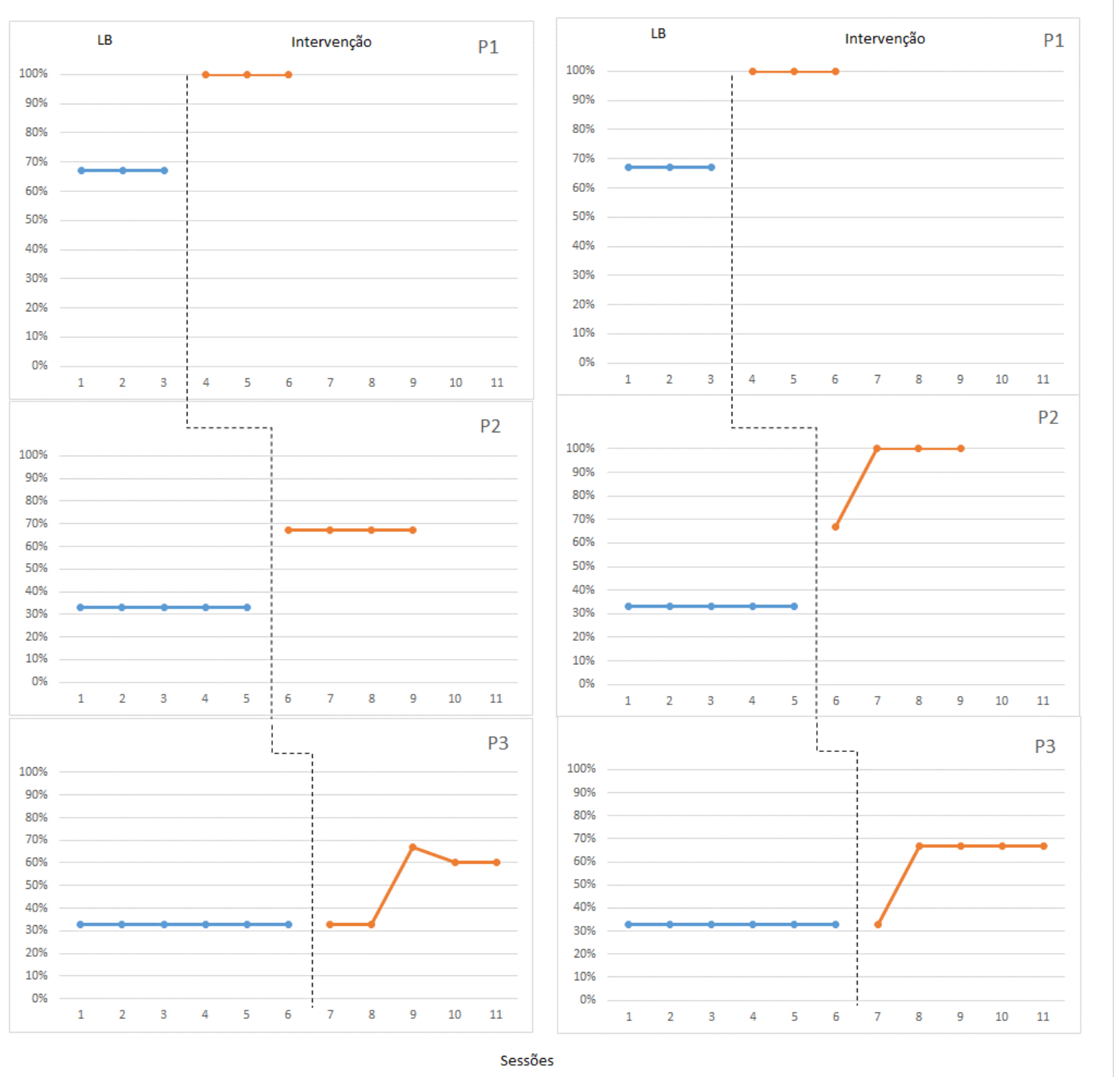

Fonte: elaboração própria, 2018.

Na Figura 2, observa-se o desempenho dos participantes com relação à habilidade de preensão palmar. Destarte, P1 obteve $67 \%$ de acertos nas sessões de linha de base. A partir da introdução da intervenção, o participante obteve um aumento de $30 \%$, atingindo uma média de 100\%, o melhor desempenho possível. Ao final, o P2 obteve, em linha de base, uma média de 33\% 
de desempenho. Após a intervenção, obteve uma média de $67 \%$, tendo um aumento de $34 \%$ da linha de base para a intervenção.

Com relação ao P3, observa-se que em linha de base atingiu 33\%. Após a intervenção atingiu uma média de $51 \%$, obtendo um aumento de $18 \%$ em relação às sessões de linha de base.

$\mathrm{Na}$ Figura 2, quanto às habilidades de velocidade do movimento, observa-se que P1 teve $67 \%$ de acertos nas sessões de linha de base. A partir da introdução da intervenção, o participante obteve um aumento de 30\%, atingindo uma média de 100\%, o melhor desempenho possível. Assim, o P2 obteve $33 \%$ em linha de base e $92 \%$ nas sessões de intervenção, atingindo um aumento de $59 \%$. Com relação ao P3, obteve um desempenho de $33 \%$ em linha de base e $60 \%$ de intervenção, um aumento de $27 \%$ em seu desempenho.

\section{DISCUSSÃO}

Na primeira variável analisada, precisão do movimento/ posicionamento, observa-se que os participantes aumentaram o nível de desempenho a partir das adaptações realizadas no jogo, favorecendo a participação de todos durante a movimentação das peças que foram mais precisas após as adaptações. Contudo, pode-se afirmar que o jogo da velha adaptado pode ser utilizado por pessoas com paralisia cerebral, afim de que estas possam ter facilidade e independência em realizar o jogo.

Com relação à amplitude do movimento, todos os participantes obtiveram melhoras nesta habilidade após as sessões com o jogo adaptado, afirmando que os efeitos do jogo adaptado foram positivos nesta habilidade, fazendo com que os jogadores pudessem conseguir desempenhar a amplitude esperada e, consequentemente, concluírem as etapas do jogo.

A preensão palmar é uma habilidade requisitada durante o jogo da velha, consequentemente, uma adaptação neste jogo, pensando nas limitações presentes na pessoa com paralisia cerebral é fundamental. Pode-se perceber neste estudo, que a partir do jogo adaptado os três participantes melhoraram esta habilidade após a intervenção. Em consonância a esta conclusão, Ferland (2006) demonstra que a interação no jogo é uma modalidade terapêutica e favorece o desenvolvimento de diversas habilidades.

A velocidade do movimento foi uma das variáveis analisadas e pode-se concluir que todos os três melhoraram no desempenho desta habilidade após a introdução da intervenção, especialmente o P2. As adaptações foram simples e puderam melhorar o desempenho nas habilidades motoras dos participantes em conformidade com a apreciação de Galvão Filho (2009), em que afirma que recursos simples e de baixo custo podem ser disponibilizados em diferentes locais para que estas pessoas possam interagir apropriadamente com variadas pessoas.

\section{CONCLUSÃO}

A partir dos resultados deste estudo foi possível demonstrar que o jogo da velha adaptado possibilitou um desempenho melhor de todos os participantes. Estes puderam segurar melhor as peças e movimentá-las mais facilmente e rapidamente, além de melhorarem os movimentos e preensão motora.

O jogo da velha adaptado poderá ser utilizado por pessoas com paralisia cerebral que apresentem diferentes limitações motoras como as dos sujeitos deste estudo, pois é um recurso simples e de baixo custo, podendo ser produzido pelos professores nas escolas, pelos pais e por qualquer pessoa. Em suma, quando pensamos na possibilidade de um recurso ser funcional e promover a independência, o jogo da velha atingiu esta finalidade. 


\section{REFERÊNCIAS}

BRASIL. Ministério da Saúde. Secretaria de Atenção à Saúde. Diretrizes Brasileira de Atenção à Pessoa com Paralisia Cerebral. Brasília: Ministério da Saúde, 2013.

BRUNO, M.M.G. Avaliação de alunos com baixa visão e múltipla deficiência na educação infantil. Dourados: Editora da UFGD, 2009, p. 198.

CAT - Comitê de Ajudas Técnicas. Ata da Reunião V, de agosto de 2007 do Comitê de Ajudas Técnicas. Brasília: Presidência da República, Secretaria Especial dos Direitos Humanos, 2007.

COZBY, P.C. Métodos de pesquisa em ciências do comportamento. Editora Altas: São Paulo, 2003.

FERLAND, F. O modelo lúdico: o brincar, a criança com deficiência e a terapia ocupacional. Editora Roca: São Paulo, 2006.

GALVÃO FILHO, T. A. Tecnologia assistiva para uma escola inclusiva: apropriação, demandas e perspectivas. 2009. 346f. Tese (Doutorado em Educação) - Faculdade de Educação, Universidade Federal da Bahia, Salvador, 2009.

LAMÔNICA, D.C.A. et al. Avaliação do aspecto semântico da linguagem em paralíticos cerebrais. Salusvita, v. 22, n. 2, p. 229-237.

LAMÔNACO, J.F.B.; CAZEIRO, A.P.M. Concepções de deficiência e reabilitação. Revista Psicologia Escolar e Educação, v.10, n.1, p.83-97, 2006. https://doi.org/10.1590/S1413-85572006000100008

PERES, R.C.N.C. O lúdico no desenvolvimento da criança com paralisia cerebral espástica. Revista Brasileira de Crescimento e Desenvolvimento Humano, São Paulo, v.14, n.3, p. 37-49, 2004. https://doi.org/10.7322/jhgd.40114

ROSENBAUM, P. et al. A report: the definition and classification of cerebral palsy april 2006. Developmental Medicine and Child Neurology, v. 49, n. 2, p. 8-14, 2007. 